\title{
Nematic wetting and filling of crenellated surfaces
}

\author{
N. M. Silvestre, ${ }^{1,2, *}$ Z. Eskandari, ${ }^{2}$ P. Patrício, ${ }^{2,3}$ J. M. Romero-Enrique, ${ }^{4}$ and M. M. Telo da Gama ${ }^{1,2}$ \\ ${ }^{1}$ Departamento de Física da Faculdade de Ciências \\ ${ }^{2}$ Centro de Física Teórica e Computacional, Universidade de Lisboa, Avenida Professor Gama Pinto 2, P-1649-003 Lisboa, Portugal \\ ${ }^{3}$ Instituto Superior de Engenharia de Lisboa, Rua Conselheiro Emídio Navarro 1, P-1959-007 Lisboa, Portugal \\ ${ }^{4}$ Departamento de Física Atómica, Molecular y Nuclear, Area de Física Teórica Universidad de Sevilla, \\ Apartado de Correos 1065, 41080 Sevilla, Spain
}

(Received 30 April 2012; published 9 July 2012)

\begin{abstract}
We investigate nematic wetting and filling transitions of crenellated surfaces (rectangular gratings) by numerical minimization of the Landau-de Gennes free energy as a function of the anchoring strength, for a wide range of the surface geometrical parameters: depth, width, and separation of the crenels. We have found a rich phase behavior that depends in detail on the combination of the surface parameters. By comparison to simple fluids, which undergo a continuous filling or unbending transition, where the surface changes from a dry to a filled state, followed by a wetting or unbinding transition, where the thickness of the adsorbed fluid becomes macroscopic and the interface unbinds from the surface, nematics at crenellated surfaces reveal an intriguingly rich behavior: in shallow crenels only wetting is observed, while in deep crenels, only filling transitions occur; for intermediate surface geometrical parameters, a new class of filled states is found, characterized by bent isotropic-nematic interfaces, which persist for surfaces structured on large scales, compared to the nematic correlation length. The global phase diagram displays two wet and four filled states, all separated by first-order transitions. For crenels in the intermediate regime re-entrant filling transitions driven by the anchoring strength are observed.
\end{abstract}

DOI: 10.1103/PhysRevE.86.011703

PACS number(s): 61.30.Dk, 61.30.Hn, 61.30.Jf

\section{INTRODUCTION}

As design and technology focus on the manipulation of materials at increasingly smaller scales, the role of surfaces and interfaces becomes increasingly important. At the micrometer scale the microscopic (nanometer) and macroscopic (millimeter) scales become entangled and new phenomena emerge. Thus, the understanding of surfaces is crucial to our ability to further miniaturize components and devices, with relevant applications to microfluidics and wetting at structured surfaces.

At the macroscopic level, wetting is described by a force balance argument, which relates the equilibrium contact angle $\theta$ of the saturated liquid droplet on the planar surface to the solid-vapor $\sigma_{\mathrm{sv}}$, solid-liquid $\sigma_{\mathrm{sl}}$, and liquid-vapor $\sigma$ surface tensions, through Young's equation:

$$
\cos \theta=\frac{\sigma_{\mathrm{sv}}-\sigma_{\mathrm{sl}}}{\sigma} .
$$

When the contact angle is zero, the liquid spreads on the surface and is said to wet the planar surface. In this context, a wetting transition occurs when the contact angle changes from a finite value to zero, as the temperature or the surface properties vary [1-4]. At the microscopic level, the wetting transition is characterized by an order parameter related to the (inverse) thickness of the liquid film in contact with the surface. In fact, at the nanometer scale, the saturated liquid droplet is accompanied by a film of adsorbed liquid, the thickness of which diverges at the wetting transition. In terms of the position of the liquid-vapor interface the wetting transition may be described as the unbinding of the fluid interface with respect to the surface [5].

\footnotetext{
*nunos@ cii.fc.ul.pt
}

More recently, a systematic investigation of simple fluids at structured surfaces was carried out, uncovering new phase transitions between dry and wet states [6]. On structured surfaces, at the macroscopic level, the effective surface area increases due to the surface structure. If the ratio of real surface area $\mathcal{S}$ to apparent (i.e., projected) area $\mathcal{A}$ is defined as the roughness parameter $r=\mathcal{S} / \mathcal{A}$, then the interfacial free energies of the solid-vapor and solid-liquid interfaces increase by the same amount, leading to

$$
\cos \theta=r \cos \theta_{\pi}
$$

where $\theta_{\pi}$ is the contact angle at the planar surface. This is known as the Wenzel equation and results from simple thermodynamic arguments [7]. Equation (2) implies that the surface structure amplifies the surface wetting properties: hydrophilic surfaces become more hydrophilic, and hydrophobic surfaces become more hydrophobic. Beyond thermodynamics, at the microscopic level, surface geometry has more drastic effects on wetting phenomena: the nature of the transition may change and new transitions, e.g., filling, may occur, preceding the wetting transitions [6].

In line with earlier work [8] we distinguish three interfacial states: dry, filled, and wet. We refer to filling as the process through which a dry state becomes filled, i.e., a mesoscopic liquid layer grows on the grooves of the surface. In this way, this mechanism can be understood as the unbending of the interface between the coexisting fluids, which remains attached to the surface [5]. On the other hand, we will refer to wetting as the process through which a dry or filled state becomes wet.

In this article we report the existence of several nematic wet and filled surface states, separated by first-order phase transitions, at crenellated (rectangular grating) surfaces. The nematic phase is characterized by long-range orientational 
order and positional disorder. The direction of molecular alignment, the nematic director $\mathbf{n}$, is arbitrary in bulk nematics but the presence of surfaces selects a particular direction, known as surface anchoring. Typical situations include homeotropic (orientation perpendicular to the surface), random planar (random orientation parallel to the surface), and degenerate planar (orientation along an arbitrary direction on the surface). Nematic wetting of planar surfaces was predicted in the framework of the Landau-de Gennes (LdG) theory [9-12] and has been observed experimentally using a variety of techniques [13-17].

Nematic wetting of structured surfaces has attracted much less attention $[18,19]$ although it is of considerable fundamental interest, with relevant applications in bistable liquid-crystal displays and microfluidic devices. The surface structure causes orientational frustration that contributes an elastic term to the free energy, which may lead to the nucleation of topological defects that will, in turn, affect the nematic order at the surface. The nematic elasticity and the nucleation of defects, with complex structure and dynamics, is a new ingredient, not present in the wetting of simple liquids at structured surfaces nor in nematic wetting at structureless ones.

Like simple fluids, nematic wetting phenomena is essentially an interplay of three possible states: dry, filled, and wet. In nematics, the filled and wet states may be characterized by a number of distinct textures or symmetries ("uniform," "symmetrically," or "asymmetrically" distorted, with or without defects, etc.) increasing the number of states the interplay of which dictates the global surface phase diagram. In most cases these are true surface states separated by (first-order) phase transitions.

In the wet state, the system has a single length scale $l$, set by the surface geometry. The elastic energy of smooth distortions is $(\mathcal{K S} / l) \tilde{\mathcal{F}}_{d}$, where $\mathcal{K}$ is the Frank elastic constant of the nematic, and $\tilde{\mathcal{F}}_{d}$ is a reduced elastic contribution to the interfacial free energy, dependent only on the surface geometry, but not on its scale $l$. The wetting transition is then given by the generalized Wenzel equation [20]

$$
1=\cos \theta=r\left(\cos \theta_{\pi}-\frac{\mathcal{K} \tilde{\mathcal{F}}_{d}}{l \sigma_{n i}}\right),
$$

where $\sigma_{n i}$ is the nematic-isotropic (NI) surface tension. In the limit of large $l$, the elastic contribution is irrelevant, and the Wenzel equation is recovered. For simple fluids, the wetting transition at structured surfaces occurs at a lower temperature or at a lower surface field than the wetting transition at flat surfaces; for nematics, the wetting thresholds may be depressed (as in simple fluids) if the geometrical structure dominates or increased if the elastic energy dominates. When the elastic energy dominates, deviations from the Wenzel equation may be significant. In the strong anchoring regime, the orientational frustration, due to the geometrical surface pattern, may lead to the nucleation of topological defects close to the surface, with a cutoff length (defect size) set by the bulk correlation length $\xi$. The presence of bulk and/or surface defects will contribute a singular $\ln (l / \xi)$ term to the rescaled elastic free energy $\tilde{\mathcal{F}}_{d}[21]$.

Beyond thermodynamics, at the microscopic level, the surface geometry has an even more profound effect on nematic wetting. As for simple fluids, it may lead to new transitions such as filling. However, the transition from a filled to a wet state may be suppressed, as the pinned NI interface in the filled state may prevent the nucleation of topological defects in the bulk nematic, which increase the elastic energy by an amount which is not compensated by the decrease in the surface anchoring energy.

In this paper we continue a systematic study of the interplay between surface geometry and the elastic energy of the distortions caused by the spatially varying surface director field. Previous studies based on the $\mathrm{LdG}$ theory were carried out for periodic triangular [20,21] and sinusoidal [22] surfaces, and general rules begin to emerge. We recall these rules and present systematic results, based on the $\mathrm{LdG}$ theory, for nematic wetting at crenellated surfaces. Preliminary results were published in [8].

Crenellated surfaces have been investigated, numerically and experimentally, in the context of zenithal bistable switching of liquid crystal (LC) devices [23,24]. These studies focused on the elastic configurations, deep in the nematic phase. Wetting transitions were not discussed as these occur at coexistence with the isotropic phase. Wetting of simple fluids on rectangular gratings was also studied in the context of capillary condensation [25]. On a capped capillary, filling of the surface turns condensation into a continuous transition, which is subsequently followed by the wetting transition. In what follows, we show that crenellated surfaces exhibit a rich nematic wetting behavior due to the presence of a large number of metastable ordered states in the wetting range of parameters. In particular, we find multiple wet and filled states, with different nematic textures. Some of the filled states are characterized by a curved or bent NI interface even for surfaces with large length scales, when compared to the nematic correlation length, while others exhibit the usual unbent interface. Thus on these surfaces filling and unbending do not occur simultaneously. In the intermediate range of surface parameters we find multiple filling and wetting transitions and re-entrant interfacial behavior, which is not observed, in general, at periodic triangular and sinusoidal surfaces.

The remainder of this paper is organized as follows. In Sec. II we summarize the results for nematic wetting of triangular and sinusoidal surfaces, setting the stage for the results on crenellated surfaces reported here. In Sec. III we describe briefly the LdG theory and the numerical method used to minimize it. In Sec. IV we present and discuss the results for the global phase diagram of nematics at crenellated surfaces. Finally, in Sec. V we summarize the theoretical results. We highlight the new results at small and intermediate roughness and contrast them with those found at triangular and sinusoidal surfaces and conclude with a brief discussion of future theoretical and experimental work.

\section{RESULTS FOR NEMATIC WETTING OF TRIANGULAR AND SINUSOIDAL SURFACES}

The LdG free energy exhibits a first-order wetting transition at structureless surfaces [12,20]. At rough surfaces (on the mesoscale) the phase behavior is, in general, richer. The surfaces investigated so far are translationally invariant (along $z$ ) 


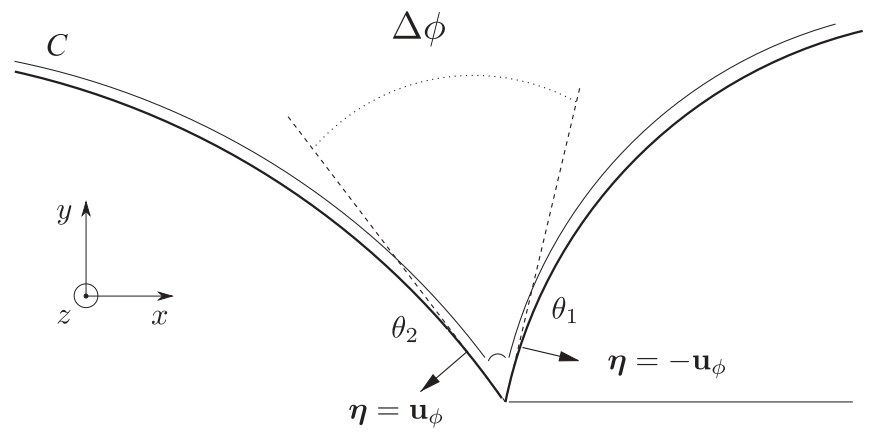

FIG. 1. Schematic representation of a surface cusp. The thick continuous line depicts the surface close to a cusp, characterized by an opening angle $\Delta \phi$. Strong anchoring conditions are enforced at the surface, with $\theta_{1}$ and $\theta_{2}$ their asymptotic values as the cusp is approached from either side. The integration contour $C$ is shown as a thin curved line, with $\eta$ its outward normal. See text for details.

with the structure defined on the $x y$ plane. These surfaces exhibit wetting behavior, which differs from that reported for simple fluids. In this section we will summarize the results. Details may be found elsewhere [8,20-22].

For triangular surfaces there is only a wetting transition [20], in agreement with the phenomenology reported for simple fluids [26]. While for simple fluids the wetting transition occurs at a lower temperature than the transition at planar surfaces, for nematics the wetting field may exceed the planar surface transition field. This is a result of the nucleation of topological defects at the surface cusps [21,27], which lead to bistability of the wetting states for opening angles close to $\alpha=\pi / 2$ (inside the groove) and an extremely slow approach to the wetting diagram predicted by the generalized Wenzel equation, Eq. (3) [8,20]. The origin of these deviations is the drastic increase of the wetting anchoring energy, even in the limit of long wavelength, due to the geometrical surface singularities [21]. The analysis for triangular surfaces can be extended straightforwardly to general cusped surfaces as follows. The leading contribution to the elastic free energy from the topological defects may be obtained from the FrankOseen free energy with a single elastic constant $\mathcal{K}$. Around each surface defect, the nematic director field $\theta$ can be written as $\theta=\theta_{0}(r, \phi)+q \phi$, where $\theta_{0}$ is a nonsingular background and $(r, \phi)$ are the radial and azimuthal polar coordinates, with origin at the defect core. Finally, $q$ is the topological charge associated to the disclination line, which depends on the nematic texture and the opening angle $\Delta \phi$ of the cusp (see Fig. 1). If we denote by $\theta_{1}$ and $\theta_{2} \equiv \theta_{1}+\Delta \theta$ the asymptotic values of the nematic director field on the surface, on either side of the cusp, as shown in Fig. 1, the topological charge can be written as $q=\Delta \theta / \Delta \phi$. Using the divergence theorem, we may obtain the elastic free energy per unit length in the $z$ direction, $f_{e l}$, as a contour integral running parallel to the surface [21]:

$$
f_{e l}=\frac{\mathcal{K}}{2} \int_{C} d s \theta(s) \eta \cdot \nabla \theta(s),
$$

where $\eta$ is the outwards normal to the integration contour. Let us focus on the contribution to $f_{e l}$ close to the cusp. The director field close to one disclination satisfies $\nabla \theta \approx q \mathbf{u}_{\phi} / r$.
In order to avoid the singularity at the cusp, the integration contour is rounded off by an arc of circle with radius of the order of the bulk correlation length $\xi$ centered at the cusp, as shown in Fig. 1. The contribution of this arc to $f_{e l}$ vanishes when $\xi$ is much smaller than the other relevant length scales as in this limit $\boldsymbol{\eta}$ is orthogonal to $\nabla \theta$. Close to the cusp, $\boldsymbol{\eta} \approx \pm \mathbf{u}_{\phi}$, as shown in Fig. 1, and $\theta$ is well approximated by the asymptotic values $\theta_{1}$ and $\theta_{2}$ on either side. Substituting these estimates into Eq. (4), the leading contribution to $f_{e l}$ close to the cusp is given by

$$
\frac{\mathcal{K}}{2} \int_{C} d s \theta(s) \eta \cdot \nabla \theta(s) \approx \frac{\mathcal{K}}{2} q \Delta \theta \ln \frac{l}{\xi}=\frac{\mathcal{K}}{2} q^{2} \Delta \phi \ln \frac{l}{\xi}
$$

where $l$ is a length which characterizes the surface periodicity. For large $l / \xi$, the leading contribution to the elastic free energy per periodic cell and unit length along the cusp axis is given by the superposition of the contributions from the different defects. As for triangular surfaces, the next-to-leading contribution to the elastic free energy is expected to depend on geometry, anchoring energy, and nematic texture, but not on the surface periodicity [21].

On sinusoidal surfaces the situation is different as the defects, if present, are necessarily bulk topological defects. It is found, in general, that filling and wetting pre-empt each other, i.e., there is a single transition-filling or wetting - from dry to a phase without topological defects. For a given roughness, the transition is either from dry-to-filled or from dry-to-wet and the sequence dry-filled-wet found in simple fluids is hardly ever observed. Whether the stable phase is the wet or the filled state depends on a combination of the surface roughness and anchoring conditions at the interfaces. The competing wet and filled phases are metastable over a relatively wide range of parameters but the nucleation of bulk topological defects-required to realize one or the other-is not compensated by a decrease in the anchoring energy in the wetting range of parameters. As a consequence, for a given surface, the sequence of filling or wetting transitions does not occur, except in a very narrow range of parameters [22].

\section{LANDAU-DE GENNES THEORY}

The nematic liquid crystal is modelled by the Landaude Gennes (LdG) free energy, which is adequate to describe inhomogeneities, including regions of reduced nematic order, on length scales of the order of the bulk correlation length, such as nematic-isotropic interfaces and topological defects. The order parameter is represented by a traceless, symmetric tensor $\mathbf{Q}$ with components:

$$
Q_{i j}=\frac{S}{2}\left(3 n_{i} n_{j}-\delta_{i j}\right)+\frac{B}{2}\left(l_{i} l_{j}-m_{i} m_{j}\right),
$$

where $n_{i}$ are the Cartesian components of the director field $\mathbf{n}$, $S$ is the nematic order parameter, which measures the orientational order along the nematic director, and $B$ is the biaxiality parameter, which measures the ordering of the molecules in directions perpendicular to $\mathbf{n}$, characterized by the eigenvectors $\mathbf{l}$ and $\mathbf{~ m}$. We consider only in-plane distortions. Although, for some systems, twist (out-of-plane) distortions may have an important role [28,29], for the LC and anchoring conditions reported in this paper these distortions are not energetically 
favorable. Therefore, we restrict this study to translationally invariant systems in the $z$ direction. Then, $\mathbf{n}=(\cos \theta, \sin \theta, 0)$, and the tensor order parameter has only three independent components, namely $Q_{x x}, Q_{y y}$, and $Q_{x y}$.

The LdG free energy may be written as

$$
\mathcal{F}=\int_{\Omega}\left(f_{\mathrm{b}}+f_{\mathrm{el}}\right) d \mathbf{x}+\int_{\partial \Omega} f_{\mathrm{s}} d s,
$$

where $f_{\mathrm{b}}$ is the bulk free-energy density, $f_{\mathrm{el}}$ is the elastic free-energy density, and $f_{\mathrm{s}}$ is the surface free energy, defined as [30]

$$
\begin{gathered}
f_{\mathrm{b}}=a \operatorname{Tr} \mathbf{Q}^{2}-b \operatorname{Tr} \mathbf{Q}^{3}+c\left[\operatorname{Tr} \mathbf{Q}^{2}\right]^{2}, \\
f_{\mathrm{el}}=\frac{L_{1}}{2} \partial_{k} Q_{i j} \partial_{k} Q_{i j}+\frac{L_{2}}{2} \partial_{j} Q_{i j} \partial_{k} Q_{i k}, \\
f_{\mathrm{s}}=-\frac{2}{3} w \operatorname{Tr}\left[\mathbf{Q} \cdot \mathbf{Q}_{\mathrm{s}}\right],
\end{gathered}
$$

where $a$ depends linearly on the temperature, $b$ and $c$ are positive constants, and $L_{1}$ and $L_{2}$ are positive parameters related to the elastic constants. If we rescale all the variables as in [31], $\tilde{\mathbf{Q}}=6 c \mathbf{Q} / b$, the positions $\tilde{\mathbf{r}}=\mathbf{r} / \xi$, where the correlation length $\xi$ is $\xi^{2}=8 c\left(3 L_{1}+2 L_{2}\right) / b^{2}$, and $\tilde{\mathcal{F}}_{\mathrm{LdG}}=$ $24^{2} c^{3} \mathcal{F}_{\mathrm{LdG}} / \xi^{3} b^{4}$, we obtain $\tilde{\mathcal{F}}=\int_{\tilde{\Omega}}\left(\tilde{f}_{\mathrm{b}}+\tilde{f}_{\mathrm{el}}\right) d \tilde{\mathbf{x}}+\int_{\partial \tilde{\Omega}} \tilde{f}_{\mathrm{s}} d \tilde{s}$, with rescaled free-energy densities:

$$
\begin{gathered}
\tilde{f}_{\mathrm{b}}=\frac{2}{3} \tau \operatorname{Tr} \tilde{\mathbf{Q}}^{2}-\frac{8}{3} \operatorname{Tr} \tilde{\mathbf{Q}}^{3}+\frac{4}{9}\left[\operatorname{Tr} \tilde{\mathbf{Q}}^{2}\right]^{2}, \\
\tilde{f}_{\mathrm{el}}=\frac{1}{3+2 k}\left(\tilde{\partial}_{l} \tilde{Q}_{i j} \tilde{\partial}_{l} \tilde{Q}_{i j}+k \tilde{\partial}_{j} \tilde{Q}_{i j} \tilde{\partial}_{l} \tilde{Q}_{i l}\right), \\
\tilde{f}_{\mathrm{s}}=-\frac{2}{3} \tilde{w} \operatorname{Tr}\left[\tilde{\mathbf{Q}} \cdot \tilde{\mathbf{Q}}_{\mathrm{s}}\right] .
\end{gathered}
$$

Here $\tau=24 a c / b^{2}$ is a dimensionless temperature, $k=L_{2} / L_{1}$ is a dimensionless elastic parameter (for stability reasons, the elastic parameter is restricted to $k>-3 / 2$ ), and $\tilde{w}=$ $16 w c / b^{2} \xi$ is the dimensionless anchoring strength. Hereafter, we will consider these rescaled variables and we will drop the tilde notation.

We set the temperature at NI coexistence, $\tau=1$, where the bulk free-energy density has two minima, corresponding to $f_{\mathrm{b}}=0$ for rescaled scalar order parameters $S_{i}=0$ (isotropic phase) and $S_{n}=1$ (nematic phase). It is important to note that the order parameter $S$ in the coexisting nematic phase is rescaled, and thus its value in real units is $b / 6 c$, which must be smaller than 1 (typically $\approx 0.4$ ). If the elastic parameter $k$ is positive (negative), the nematic aligns parallel (perpendicular) to the nematic-isotropic interface. Finally, $\mathbf{Q}_{\mathrm{s}}$ defines the preferred tensor order parameter at the surface. We consider homeotropic alignment by setting $\mathbf{Q}_{\mathrm{s}}=(3 \boldsymbol{v} \otimes \boldsymbol{v}-1) / 2$, with $v$ the normal to the surface, in line with previous work $[9,10,12]$.

\section{CRENELLATED SURFACES}

We consider crenellated surfaces (see Fig. 2) that favor perpendicular (homeotropic) alignment of the nematic. The surface is translationally invariant in the $z$ direction and has a periodic rectangular cross section, characterized by three lengths: the separation between crenels (top plateau) $l_{1}$, the width $l_{2}$, and the depth $h$ of the crenels. The periodic structure

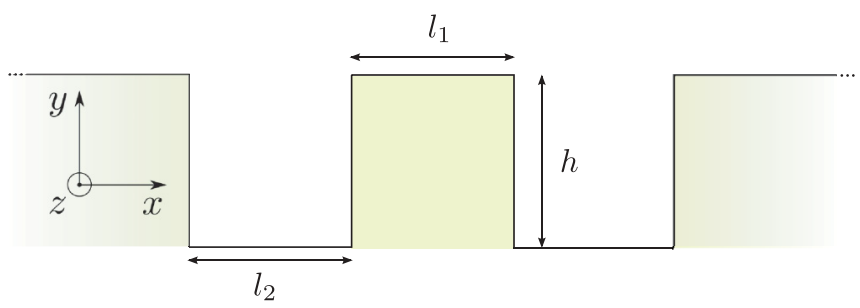

FIG. 2. (Color online) Crenellated surfaces. $l_{1}$ is the separation between crenels, and $l_{2}$ and $h$ are the width and depth of the crenels. The structure is periodic with wavelength $\lambda=l_{1}+l_{2}$.

has wavelength $\lambda=l_{1}+l_{2}$. In what follows, lengths will be measured in units of the correlation length $\xi$ of the nematic. The use of a mesoscopic theory, such as the Landau-de Gennes theory, requires that the surface characteristic lengths are at least one order of magnitude larger than the bulk correlation length. We set $k=2$, which implies that the nematic alignment is parallel to the NI interface (if there is one), away from the surface boundaries.

The roughness of the surface depends on the ratios $l_{1} / l_{2}$ and $h / l_{2}, r \equiv r\left(l_{1} / l_{2}, h / l_{2}\right)$, and is given by $r=1+2 h /\left(l_{1}+l_{2}\right)$. At fixed ratio of the separation and width of the crenels (ratio of the top and bottom plateaux), $l_{1} / l_{2}$, the roughness increases with the ratio of the depth and width of the crenels, $h / l_{2}$. For a given surface geometry, we calculate the free energy of the interfacial states: dry $(D)$, filled $(F)$, and wet $(W)$, using the anchoring strength as the control parameter. At fixed anchoring strength, nematic filled and wet states exhibit, in general, distinct director configurations or textures (see Fig. 3), which are investigated using different initial conditions.

In addition to the dry state [Fig. $3(D)$ ], we have found a uniform [Fig. $\left.3\left(W_{u}\right)\right]$ and two distorted wet states distinguished by the symmetry of the nematic director. The uniform wet state is the planar surface limit, roughness $r=1$, where the nematic texture is uniform and perpendicular to the surface. At low roughness, the nematic director bends symmetrically around the bottom corners of the crenel [Fig. $3\left(W_{d}^{s}\right)$ ], in order to follow the homeotropic anchoring favored by the surface everywhere. This distortion is more evident at high anchoring strengths where the conflicting orientations near the corners lead to the nucleation of topological defects that adopt a symmetric configuration. When the roughness increases further the nematic takes a distorted asymmetric texture [Fig. 3 $\left.\left(W_{d}^{a}\right)\right]$ which is bistable, as the director bends around one of the bottom corners and splays around the other.

We have also found distinct filled states. At intermediate roughness, the filled state has a NI interface that is curved, or bent, inside the crenels [Fig. $3\left(F_{b}\right)$ ], while at rough surfaces the NI interface is flat, or unbent, at the top of the crenels [Fig. $3\left(F_{u}\right)$ ]. In the unbent filled state, the nematic director far from the bottom of the crenels is perpendicular to the lateral surfaces, in line with the orientation favored by the flat NI interface. Near the bottom, however, the nematic is distorted as a result of the homeotropic anchoring favored by the surfaces. This state is bistable as the nematic textures may be bent towards the left or the right bottom corner (and splayed around the other).

Under strong anchoring conditions a nematic droplet or film nucleates on the upper horizontal surfaces, corresponding to 
(D)
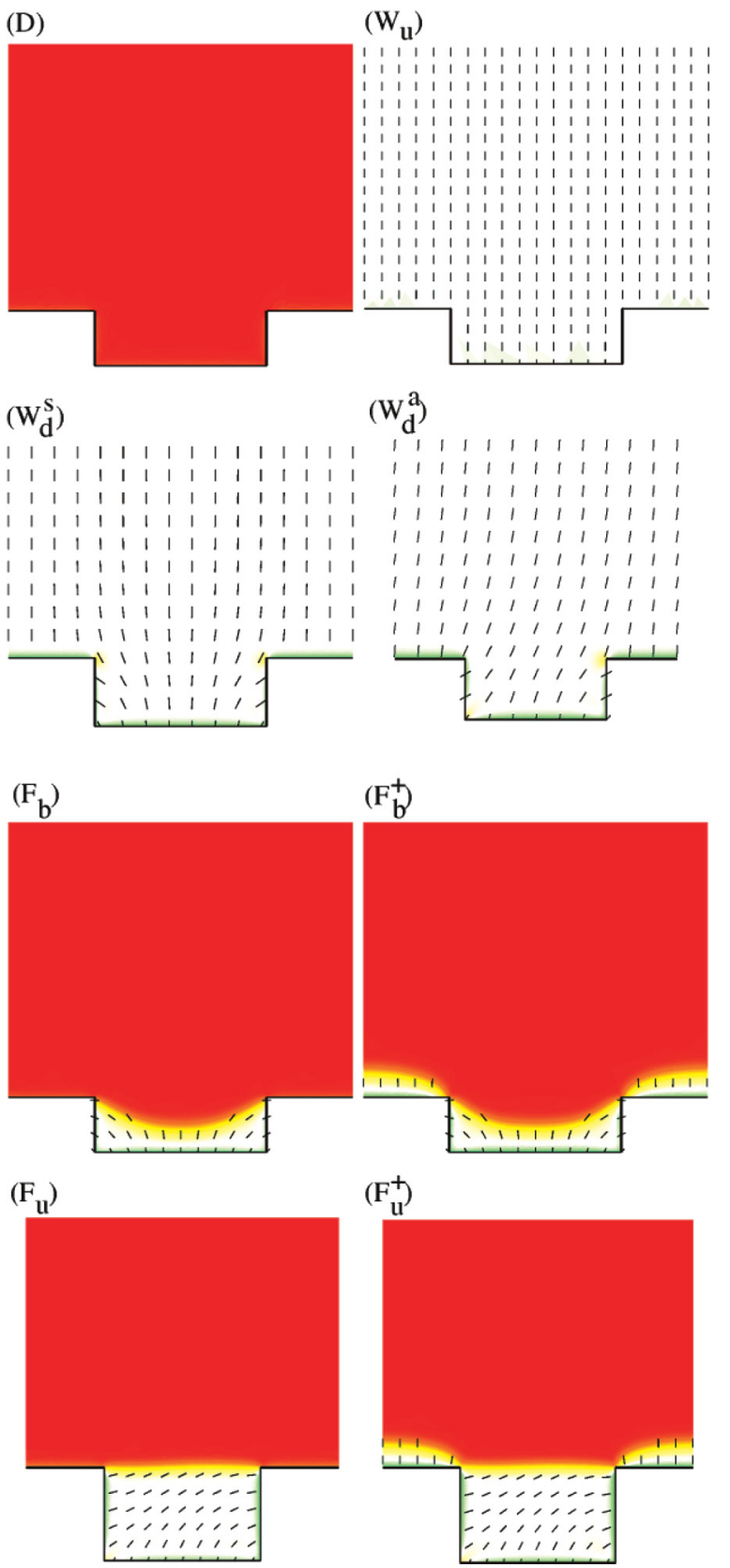

FIG. 3. (Color online) Interfacial states of crenellated surfaces for nematics with positive elastic anisotropy. $(D)$ dry state, $\left(W_{u}\right)$ uniform wet state, $\left(W_{d}^{s}\right)$ symmetric wet state, $\left(W_{d}^{a}\right)$ asymmetric wet state, $\left(F_{b}\right)$ bent filled state, $\left(F_{b}^{+}\right)$bent filled state with droplets, $\left(F_{u}\right)$ unbent filled state, $\left(F_{u}^{+}\right)$unbent filled with droplets.

new filled states [Fig. $3\left(F_{b}^{+}\right)$and $\left(F_{u}^{+}\right)$], with bent and unbent NI interfaces. The unbent state is also bistable.

\section{A. Crenel width $=$ crenel separation}

We start by considering a surface where the width of the crenels is equal to their separation, $l_{1}=l_{2}=l$. Figure 4 illustrates the global phase diagram for $l=10 \xi$. The lines represent first-order transitions between interfacial states (see Fig. 3). At low and high roughness, corresponding to shallow and deep crenels, the phase diagram resembles that of sinusoidal surfaces [22]. For low roughness, when the crenels are shallow,

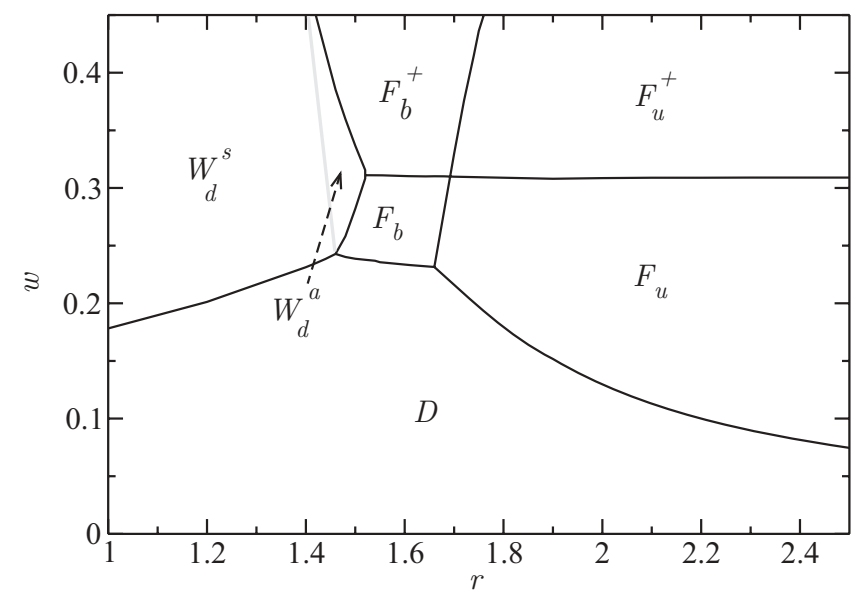

FIG. 4. Phase diagram for a crenellated surface with the same crenel width and separation, $l 1=l 2=10 \xi$. The lines correspond to first-order phase transitions that separate dry $(D)$, wet $(W)$, and filled $(F)$ states. Two distinct wet states and four distinct filled states are observed. At the wetting transition, the nearly uniform wet state $\left(W_{u}\right)$ at low roughness changes continuously to a symmetric distorted wet state $\left(W_{d}^{s}\right)$ as the anchoring increases. There is a bistable asymmetric nematic wet state $\left(W_{d}^{a}\right)$ at intermediate roughness. The wet states are separated by a weak first-order transition indicated by the grey line. There are also filled states, characterized by a bent $\left(F_{b}\right)$ or unbent NI interface $\left(F_{u}\right)$. The unbent filled states are bistable. At strong anchoring the top surfaces promote the nucleation of nematic droplets or films for bent $\left(F_{b}^{+}\right)$and unbent $\left(F_{u}^{+}\right)$filled states. See Fig. 3 for a sketch of the corresponding states.

the nematic undergoes a wetting transition to a symmetric wet state $W_{d}^{s}$. At the transition the distortions are small, and become more pronounced as the anchoring strength increases. These distortions vanish in the limiting case of a planar surface, where the nematic takes a uniform homeotropic alignment. For intermediate values of the roughness the nematic deformations at the transition become more pronounced and increasing the anchoring strength leads to a configurational transition from $W_{d}^{s}$ to the asymmetric wet state $W_{d}^{a}$. This transition is weakly first order, and we could not resolve the crossing of the two branches with the current numerical technique. We have, however, estimated the transition, depicted in grey in the phase diagrams, by inspection of the nematic textures.

In the limit of highly rough surfaces, when the crenels are deep, a filling transition is observed to a state where the nematic fills the crenels, $F_{u}$. The NI interface is flat and is pinned at the crenels top corners. This state is characterized by a uniform parallel director near the NI interface which bends asymmetrically near the bottom surface. This configuration is bistable as the nematic director can bend in two equivalent ways.

At intermediate roughness, however, when the width of the crenels $l$ is similar to their their depth $h$, the surface exhibits a novel filling transition, where the NI interface remains bent, $F_{b}$, with a symmetric nematic texture inside the crenels. These bent nematic filled states were not observed at triangular or sinusoidal surfaces.

In both filled states, $F_{b}$ or $F_{u}$, the top surfaces promote the nucleation of nematic droplets (or films) as the anchoring 
strength increases, through the mechanism that drives wetting on planar surfaces. On crenellated surfaces, however, the NI interface remains pinned at the top corners to prevent the nucleation of topological defects, the energy of which is not compensated by depinning the NI interface. As a result, the wetting transition is pre-empted. Recent Lattice Boltzmann calculations indicate that it is possible to switch between bent and unbent filled states through the coupling to weak external electric fields [32].

\section{Wetting transition, $D \leftrightarrow W_{u}$}

Structured surfaces with low roughness and weak anchoring induce smooth deformations of the nematic director field. As the anchoring increases a wetting transition takes place, in line with the generalized Wenzel equation (3). This equation has been tested for sinusoidal and triangular periodic surfaces and it was found to agree with the LdG theory when the logarithmic contributions from the topological defects nucleated at the triangular surface singularities are taken into account [21].

At low roughness $(h \ll l)$ and weak anchoring $(w \ll 1)$, crenellated surfaces are wetted by a nearly uniform (weakly distorted) wet state as the gain in elastic energy that results from the (nearly) uniform texture compensates the cost of the nematic misalignment at the lateral surfaces. As the anchoring strength increases, the (nearly) uniform wet state changes smoothly into a symmetric distorted wet state $W_{d}^{s}$.

The free energy per unit length and unit cell, $f=\mathcal{F} /\left(L_{z} n\right)$, where $L_{z}$ is the length of the system perpendicular to the surface, and $n$ is number of periodic cells, in the dry state [Fig. 3 $(D)]$ is proportional to the isotropic fluid-surface interfacial tension $\sigma_{i s}$ and is given by

$$
f^{(D)}=\sigma_{i s}\left(l_{1}+l_{2}+2 h\right)=\sigma_{i s} r\left(l_{1}+l_{2}\right),
$$

where $r$ is the roughness parameter. The free energy of the (nearly) uniform wet state is a weighted sum of the NI $\sigma_{n i}$ and the nematic-surface $\sigma_{n s}$ interfacial tensions. Nematic interfacial free energies depend on the nematic orientation, and thus we write

$$
\begin{aligned}
f^{\left(W_{u}\right)} & =\left(\sigma_{n i}^{\|}+\sigma_{n s}^{\perp}\right)\left(l_{1}+l_{2}\right)+2 \sigma_{n s}^{\|} h, \\
& =\left(\sigma_{n i}^{\|}+\sigma_{n s}^{\perp}+\sigma_{n s}^{\|}(r-1)\right)\left(l_{1}+l_{2}\right) .
\end{aligned}
$$

The wetting transition occurs when $f^{(D)}=f^{\left(W_{u}\right)}$, resulting in the balance equation

$$
\left(\sigma_{n s}^{\perp}-\sigma_{i s}\right) r+\left(\sigma_{n s}^{\|}-\sigma_{n s}^{\perp}\right)(r-1)+\sigma_{n i}^{\|}=0 .
$$

The second term depends on the anisotropy of the surface potential, which favors a particular nematic orientation. In the weak anchoring regime the first two terms can be calculated (see Appendix A) yielding $\sigma_{n s}^{\perp}-\sigma_{i s}=-w+O\left(w^{3}\right)$ and $\sigma_{n s}^{\|}-\sigma_{n s}^{\perp}=3 w / 2+O\left(w^{2}\right)$. Consequently, the wetting transition occurs when

$$
w_{t}=\frac{2 \sigma_{n i}^{\|}}{3-r} .
$$

This implies that (nearly) uniform wet states [Fig. $\left.3\left(W_{u}\right)\right]$ do not occur at very rough surfaces, $r \geqslant 3$. Within the LdG theory the NI interfacial tension $\sigma_{n i}^{\|}$is [20]

$$
\sigma_{n i}^{\|}=\frac{1}{6} \sqrt{\frac{6+k}{3+2 k}},
$$

which may be used in Eq. (17).

\section{Uniform to distorted wetting transition $W_{u} \leftrightarrow W_{d}$}

In general, structured surfaces with homeotropic anchoring promote smoothly distorted wet states, where the nematic orientation follows the surface structure. At crenellated surfaces, however, the presence of geometrical singularities (corners) implies that nematic configurations which follow the surface anchoring will have a number of topological defects. For shallow crenels, $W_{u}$, the uniform wet state analyzed in the previous Sec. IV A1, as an approximation to the nearly uniform wet state, is energetically favorable. As the depth of the crenels increases, the contribution from the surface alignment at the lateral surfaces increases and induces significant distortions. As in periodic triangular surfaces [20,21], the free energy of a distorted wet configuration may be analyzed using the arguments outlined in Sec. II. In each cell, the corners of the crenellated surface generate topological defects with charge $\pm 1 / 3$ at the top (with opening angle $\Delta \phi=3 \pi / 2$ ), and \pm 1 at the bottom (with opening angle $\pi / 2$ ) of the crenels. Using Eq. (4), the free energy per unit length and cell of the distorted wet state may be written:

$$
\begin{aligned}
f^{\left(W_{d}\right)}= & \sigma_{n s}^{\perp}\left(l_{1}+l_{2}+2 h\right)+\sigma_{n i}^{\|}\left(l_{1}+l_{2}\right) \\
& +\frac{2 \pi \mathcal{K}}{3} \ln \frac{h}{\xi}+B\left(w, \frac{l_{1}}{h}, \frac{l_{2}}{h}\right),
\end{aligned}
$$

where $\mathcal{K}(k)=(9 / 2)(2+k) /(3+2 k)$ is an effective elastic constant [21]. The second-to-last term accounts for the leading contribution to the elastic free energy associated with the topological defects. $B$ corresponds to the next-to-leading order contribution to the elastic free energy which, as discussed in Sec. II, is expected to depend on the anchoring strength $w$ and the ratios of the relevant length scales of the surface structure, but not on the surface scale. In addition, this contribution will be different for symmetric wet $W_{d}^{s}$ and asymmetric wet $W_{d}^{a}$ states, by contrast to the leading contribution which is independent of the texture. In order to check this scaling, we subtracted the surface tension and logarithmic contributions to the free energy $f$ obtained from the $\mathrm{LdG}$ theory, for different cell sizes keeping the aspect ratios fixed. As expected, we observe a collapse for moderate values of the anchoring strength $w$. The collapse occurs at smaller values of $w$ as the depth of the crenels $h$ increases (see Fig. 5).

The transition between the two wet states $W_{u}$ and $W_{d}$ is given by the free-energy balance $f^{\left(W_{u}\right)}=f^{\left(W_{d}\right)}$, and yields

$$
\begin{aligned}
2 h\left(\sigma_{n s}^{\|}-\sigma_{n s}^{\perp}\right) & \approx 3 w h \\
& =\frac{2 \pi \mathcal{K}}{3} \ln \frac{h}{\xi}+B\left(w, \frac{l_{1}}{h}, \frac{l_{2}}{h}\right) .
\end{aligned}
$$

In deep crenels, large $h / l_{2}$, we may neglect $B$. Then, the distorted wet state is stable for sufficiently strong anchoring, $w$, in deep crenels, $h$. The balance equation also implies that 


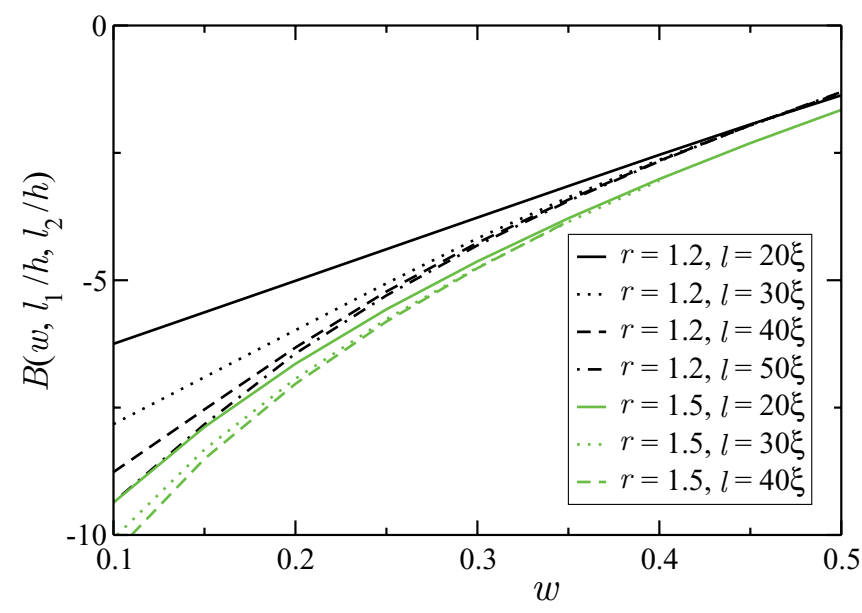

FIG. 5. (Color online) Scaling of the free energy, $\mathcal{F}-\sigma_{n w}^{\perp}\left(l_{1}+\right.$ $\left.l_{2}+2 h\right)-(2 \pi / 3) \mathcal{K} \ln (h / \xi)$ of distorted wet states as a function of the anchoring strength $w$ with the system size, for roughness $r=1.2$ and $r=1.5 . \mathcal{K}(k)$ is an effective elastic constant. The curves for equal roughness collapse at moderate to high values of the anchoring strength. The collapse occurs at lower values of the anchoring as the depth of the crenels $h$ increases.

by increasing the scale of the system (by increasing both $l$ and $h$ ), the left-hand side increases linearly, while the righthand side increases logarithmically. As a result, distorted wet states are favored with respect to (nearly) uniform wet states in larger systems. We note, however, that the analysis does not discriminate between symmetric $W_{d}^{s}$ and asymmetric $W_{d}^{a}$ wet states. A symmetric distortion implies a configuration with topological defects with the same charge on the left and right corners: $-1 / 3$ at the top, and +1 at the bottom corners, as depicted in Fig. $3\left(W_{d}^{s}\right)$. By contrast, the asymmetric distorted wet state is characterized by $+1 / 3$ and $-1 / 3$ charges at the top and -1 and +1 at the bottom corners, as depicted in Fig. 3 $\left(W_{d}^{a}\right)$. The free energy of the distorted states has the same logarithmic contribution, as this does not depend on the sign of the topological charges, but the next-to-leading contribution is different. Our detailed calculations suggest that, in general, the free energy is lower for the $W_{d}^{s}$ state, which is thus the stable distorted state. As mentioned previously, we have not observed a transition between the nearly uniform wet state and the distorted symmetric wet state. However, the fact that in a small region of the phase diagram (Fig. 4) the $W_{d}^{a}$ state is stable indicates that next-to-leading contribution $B$ is indeed different for each wet state and strongly dependent on the geometric parameters and the anchoring strength.

\section{Filling transition to the unbent state $D \leftrightarrow F_{u}$}

We consider now the limit of high roughness (Fig. 4), which corresponds to surfaces with deep crenels. In this regime, we have found a filling transition, from the dry $(D)$ to a filled state characterized by a flat or unbent NI interface $\left(F_{u}\right)$. In deep crenels, the nematic is distorted only at the bottom. Elsewhere, the nematic configuration is uniform, perpendicular to the lateral surfaces and parallel to the NI interface. Neglecting the elastic contributions from the distortions, we can write the free energy per unit length and cell as

$$
\begin{aligned}
f^{\left(F_{u}\right)} & =\sigma_{i s} l_{1}+\left(\sigma_{n i}^{\|}+\sigma_{n s}^{\|}\right) l_{2}+2 \sigma_{n s}^{\perp} h, \\
& =\left(\sigma_{i s}-\sigma_{n s}^{\perp}\right) l_{1}+\left(\sigma_{n s}^{\|}-\sigma_{n s}^{\perp}\right) l_{2}+\sigma_{n s}^{\perp} r\left(l_{1}+l_{2}\right)+\sigma_{n i}^{\|} l_{2} .
\end{aligned}
$$

The filling transition occurs when $f^{(D)}=f^{\left(F_{u}\right)}$, which leads to the balance equation

$$
\left(\sigma_{n s}^{\perp}-\sigma_{i s}\right)\left(\frac{l_{1}}{l_{2}}(r-1)+r\right)+\sigma_{n s}^{\|}-\sigma_{n s}^{\perp}+\sigma_{n i}^{\|}=0,
$$

the solution of which is

$$
w_{t}=\frac{\sigma_{n i}^{\|}}{\frac{l_{1}}{l_{2}}(r-1)+r-\frac{3}{2}} .
$$

By contrast to the wetting transition $D \leftrightarrow W_{u}$, the filling transition to the unbent state depends not only on the surface roughness $r$ but also on the ratio between the crenel separation and width, $l_{1} / l_{2}$. Equation (23) implies that, at fixed $l_{1} / l_{2}$, the filling transition to the unbent state occurs only at surfaces with roughness above a certain threshold, $r>\left(l_{1} / l_{2}+3 / 2\right) /\left(l_{1} / l_{2}+1\right)$.

\section{Filling transition to the bent state $D \leftrightarrow F_{b}$}

The filling transition predicted by Eq. (23) does not account for the nematic distortions that are, of course, induced by the geometric structure of the surface. The elastic energy arising from these distortions is not easy to take into account analytically, as discussed previously. Here we give a crude description of the bent filled state.

In shallow crenels the bent filled state is approximated by two circular domains of nematic bent around the bottom corners, with a boundary that is circular of radius $h$, connected by a flat layer of uniform homeotropic nematic, of height $h_{f}<h$ (see Fig. 6). As the crenel depth increases the two circular domains increase, and the uniform flat layer that connects them decreases.

We approximate the free energy per unit length and cell of this state by

$$
\begin{aligned}
f^{\left(F_{b}\right)} \approx & \sigma_{n s}^{\perp}\left(2 h+l_{2}\right)+\sigma_{i s} l_{1}+\sigma_{n i}^{\|}\left(\pi h-2 h_{f}\right) \\
& +\sigma_{n i}^{\perp}\left(l_{2}-2 \sqrt{h^{2}-h_{f}^{2}}\right)+f_{e}(h),
\end{aligned}
$$

where the height of the interface is, determined by minimization, $h_{f}=\sigma_{n i}^{\|} / \sqrt{\sigma_{n i}^{\perp^{2}}+\sigma_{n i}^{\|^{2}}} h . f_{e}(h)$ is the elastic free energy per unit length and cell associated with the director deformations. For simplicity, we assume that these arise from the circular domains centered at the bottom corners, which correspond to +1 topological surface defects. Within the

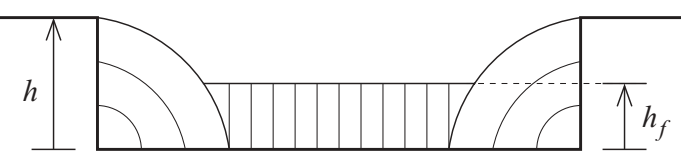

FIG. 6. Bent filled state approximated by two circular domains, of radius $h$, of nematic bent around the bottom corners connected by a flat layer of uniform homeotropic nematic, of height $h_{f}$. 
Frank-Oseen elastic theory $f_{e}(h)$ is given by

$$
f_{e}(h)=\frac{\pi \mathcal{K}}{2} \ln \frac{h}{\xi}+B\left(w, \frac{h}{l_{1}}, \frac{h}{l_{2}}\right) .
$$

The filling transition occurs when the dry and the bent filled states coexist, $f^{(D)}=f^{\left(F_{b}\right)}$, which occurs at

$$
w_{t}=\frac{\sigma_{n i}^{\|}\left(\pi \frac{h}{l_{2}}-2 \frac{h_{f}}{l_{2}}\right)+\sigma_{n i}^{\perp}\left(1-2 \frac{\sqrt{h^{2}-h_{f}^{2}}}{l_{2}}\right)+f_{e}(h) / l_{2}}{\frac{l_{1}}{l_{2}}(r-1)+r} .
$$

This transition may occur at surfaces with roughness $r>$ $1 /\left(1+l_{1} / l_{2}\right)$, which is lower than the roughness threshold of the transition to the unbent state obtained in Sec. IV A3. The analysis also shows that the bent filled state is stabilized by wider crenels, i.e., large $l_{2}$.

\section{Transition between filled states with and without nematic droplets $\boldsymbol{F}_{\boldsymbol{u}(b)} \leftrightarrow \boldsymbol{F}_{u(b)}^{+}$}

When the stable configuration is a filled state, increasing the surface anchoring $w$ promotes the nucleation of nematic droplets on the top surfaces, suggesting the growth of a wetting layer. In order to prevent the nucleation of topological defects, however, the NI interface stays pinned at the top corners, and does not detach from the surface. Figure 7 depicts the anchoring strength $w_{t}$, at the transition-which is discontinuous - as a function of the crenel separation $l_{1}$. It decreases rapidly at small crenel separations, and decays slowly towards a saturation value at large crenel separations.

In the range of crenel separation $l_{1}$, which was investigated, the nematic director in the droplets is uniform and homeotropic everywhere, including at the NI boundary (which is planar at the free NI interface). The pinning at the corners does not allow the NI interface to detach and the two interfaces interact strongly, with the surface anchoring prevailing at the bound NI

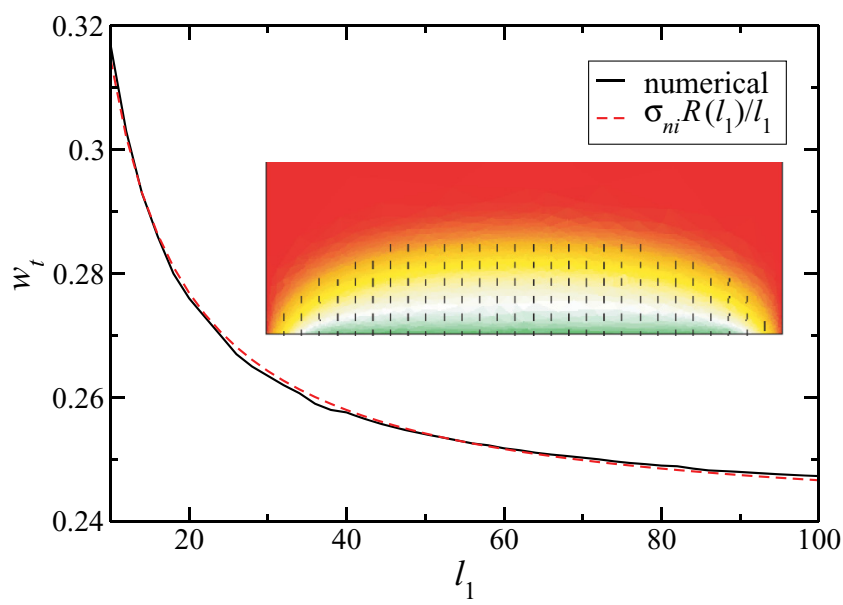

FIG. 7. (Color online) Filling $F_{u(b)}$ to filling $F_{u(b)}^{+}$transition. Anchoring strength $w_{t}$ as a function of the crenel separation $l_{1}$. The full-black line corresponds to the anchoring strength $w_{t}$ obtained numerically. The dashed-red line is the analytical estimate $w_{t}=$ $\sigma_{n i}^{\perp} R\left(l_{1}\right) \xi / l_{1}$, where $R\left(l_{1}\right)=l_{1} / \xi+4+O\left(1 / l_{1}\right)$. The inset depicts the nematic droplet on the top surface. The nematic director is strongly anchored to the surface and is homeotropic everywhere. interface. The shape of these uniformly aligned droplets, $z(\mathbf{x})$ can be calculated analytically using an effective Hamiltonian (see Appendix B).

At large crenel separations $l_{1}$, the shape of the droplet $z(x)$ is given by

$$
z(x) \approx \sqrt{2} \xi \ln \left(\frac{l_{1}}{\pi} \sqrt{\frac{w}{\sigma_{n i}^{\perp} \xi^{2}}} \sin \frac{\pi x}{l_{1}}\right) .
$$

This indicates that the droplet height increases logarithmically with $l_{1}$ up to the midpoint of the top surface, where it attains a maximum given by $z\left(l_{1} / 2\right) \simeq \sqrt{2} \xi \ln l_{1}+O(1)$. As $l_{1}$ increases the droplets spread and become flatter and flatter resembling a film, the thickness of which diverges logarithmically with $l_{1}$.

The free energy per unit length and unit cell of the droplets, in the limit of large $l_{1}$, is given by Eq. (B11). At the transition this free energy is equal to the free energy of the state without a droplet, $\sigma_{i s} l_{1}$, yielding the anchoring transition strength $w_{t}$,

$$
w_{t}=\sigma_{n i}^{\perp}+4 \sigma_{n i}^{\perp} \frac{\xi}{l_{1}}+O\left(\frac{1}{l_{1}^{2}}\right) .
$$

This estimate for $w_{t}$ is compared to the numerical results of the LdG free energy in Fig. 7. The agreement is excellent for the whole range of crenel separations.

\section{Increasing the size of the system}

Increasing the size of the system to $l=20 \xi$ results in a phase diagram similar to that depicted in Fig. 4. Figure 8 illustrates the differences between the two. At low roughness, the wetting transition $D \leftrightarrow W_{u}$ is unaffected by the scale of the system, as expected. For rough surfaces the filling transition $D \leftrightarrow F_{u}$ converges to the same asymptotic value, which is also scale independent. The transition from dry to a bent filled state $D \leftrightarrow F_{b}$ is stabilized and is observed at lower roughness and anchoring strength. Likewise, the transitions between filled states with and without nematic droplets on the top surfaces, $\left(F_{u(b)} \leftrightarrow F_{u(b)}^{+}\right)$, occur at lower values of the

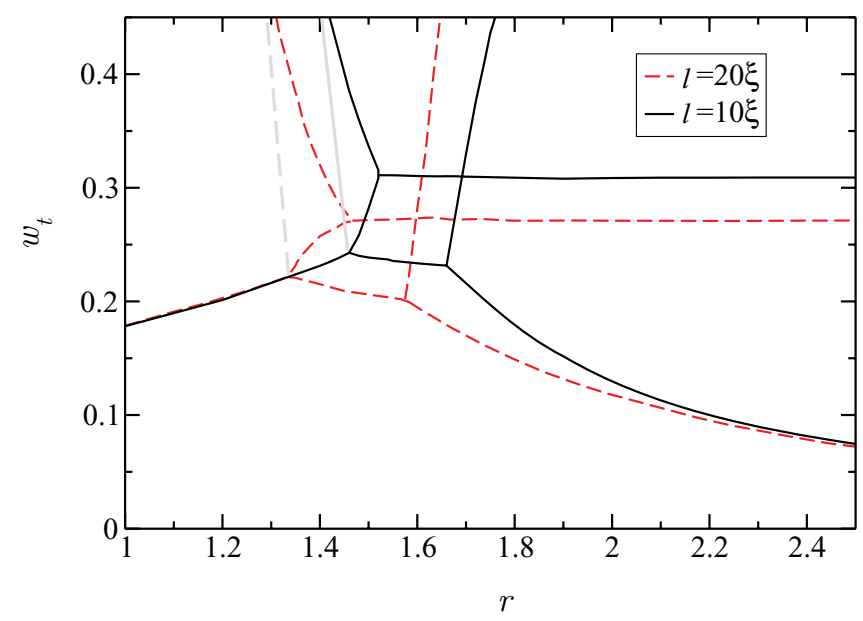

FIG. 8. (Color online) Phase diagrams for surfaces with $l_{1}=l_{2}=$ $10 \xi$ (full-black line) and $20 \xi$ (dashed-red line). Re-entrant filling is enhanced. The grey lines represent the weak first-order transition between the two wet states (see Fig. 4). 


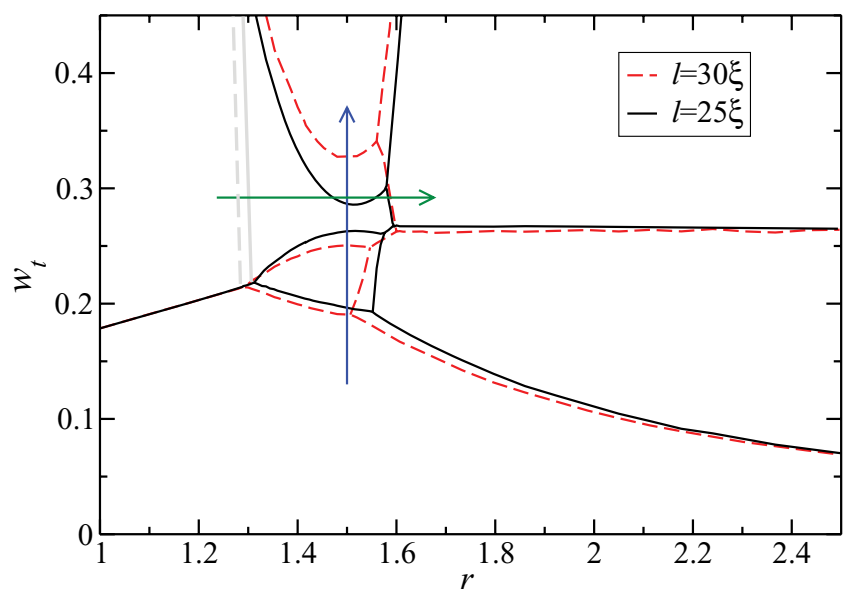

FIG. 9. (Color online) Phase diagrams for systems with $l_{1}=l_{2}=$ $25 \xi, 30 \xi$. At low and high roughness the phase diagram is similar to the previous ones. At intermediate roughness, there is a clear enhancement of the re-entrant regions, as the wet state is stable between the filled states $F_{b}$ and $F_{b}^{+}$. The grey lines represent the weak first-order transition between the two wet states (see Fig. 4). Blue (vertical) and green (horizontal) arrows indicate re-entrant paths by varying the anchoring and the roughness, respectively.

anchoring strength, in line with the discussion of the previous section. As a result, the region where re-entrant filling is observed increases.

As the size of the system increases further, the phase diagram changes significantly. Figure 9 depicts the phase diagrams for systems with $l / \xi=25,30$. The low and high roughness regimes exhibit the same behavior, as they are independent of the system size. The most striking changes occur at intermediate roughness. There is a clear enhancement of the filled states at intermediate roughness. The re-entrant region is also more prominent as the wet state is clearly separated from the filled states, with and without nematic droplets, $F_{b}$ and $F_{b}^{+}$. In this regime, there is a transition from the filled to the wet state, $F_{b} \leftrightarrow W_{d}$, at low anchoring strength, without the nucleation of topological defects. As the anchoring increases, the nematic distortions become stronger leading to the nucleation of defects. As a result the system re-enters the filled state-with nematic droplets - through the pinning of the NI interface at the top corners of the crenels, Fig. $3\left(F_{b}^{+}\right)$. For large system sizes there is a second narrow region, at higher roughness, where the re-entrant sequence dry $\rightarrow$ filled $\rightarrow$ wet $\rightarrow$ filled is observed. The filled states, however, are unbent filled states $F_{u}$.

In a narrow range of the anchoring strength, increasing the roughness results in geometrically induced re-entrant sequences where the system goes through wet $\rightarrow$ (bent) filled $\rightarrow$ wet $\rightarrow$ (unbent) filled states, however, are unbent filled states $F_{u}$.

In a narrow range of the anchoring strength, increasing the roughness results in geometrically induced re-entrant sequences where the system goes through wet $\rightarrow$ (bent) filled $\rightarrow$ wet $\rightarrow$ (unbent) filled states, a sequence that is not observed for simple fluids and appears to be a feature of nematic liquid crystals at crenellated surfaces.

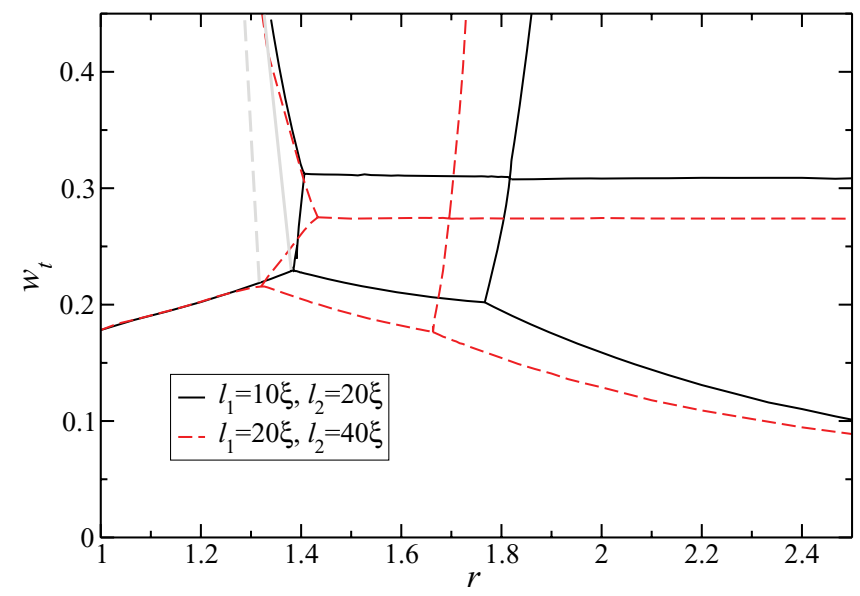

FIG. 10. (Color online) Phase diagrams for systems with $l_{1}=$ $10 \xi, l_{2}=20 \xi$ and $l_{1}=20 \xi, l_{2}=40 \xi$. The stability of the bent filled, $F_{b}$ and $F_{b}^{+}$, is enhanced. The grey lines represent the weak first-order transition between the two wet states (see Fig. 4).

\section{B. Crenel width $\neq$ crenel separation}

Distinct phase diagrams are obtained when the separation between crenels is different from their width. In this section we address crenellated surfaces where $l_{1} \neq l_{2}$. We consider two cases: (1) wide or closely spaced crenels $l_{2}=2 l_{1}$, and (2) narrow or widely separated crenels $l_{1}=2 l_{2}$.

\section{Wide or closely spaced crenels: $l_{2}=2 l_{1}$}

When the width of the crenel is twice the crenel separation, the stability of the bent filled state increases, i.e., bent filled states occur in a wider range of parameters. The phase diagrams for $l_{1}=10 \xi, l_{2}=20 \xi$ and $l_{1}=20 \xi, l_{2}=40 \xi$ are illustrated in Fig. 10. The enhanced stability of the symmetric filled states results from the fact that in wide crenels bending of the NI is less severe and the corresponding elastic energy is lower. As the depth of the crenels increases the NI interface becomes flat or unbent. We note that the transitions to unbent filled state occur at a higher roughness $r>4 / 3$. As the system size increases the stability of the bent filled states also increases, as they occur at lower roughness and anchoring strength. Otherwise, the phase diagram resembles that of small systems with $l_{1}=l_{2}$.

\section{Narrow or widely separated crenels: $l_{1}=2 l_{2}$}

Finally, we consider narrow or widely separated crenels, on surfaces where the distance between crenels is twice their width. Figure 11 compares the phase diagrams for systems with $l_{1}=20 \xi, l_{2}=10 \xi$ and $l_{1}=40 \xi, l_{2}=20 \xi$. In this case, the stability of the bent filled states is reduced, i.e., bent filled states occur in a smaller range of parameters. By contrast, the transition to unbent filled states with flat NI interfaces occurs at lower roughness $r>7 / 6$. Wetting is also enhanced, and as a consequence, the re-entrant region becomes more prominent. The phase diagram resembles that of large systems with $l_{1}=l_{2}$ (same width and separation) in the sense that the wet region intrudes into the filled regions. In this case, the bent filled states-without nematic droplets - are reduced to small pockets or islands of stability that seem to vanish for the 


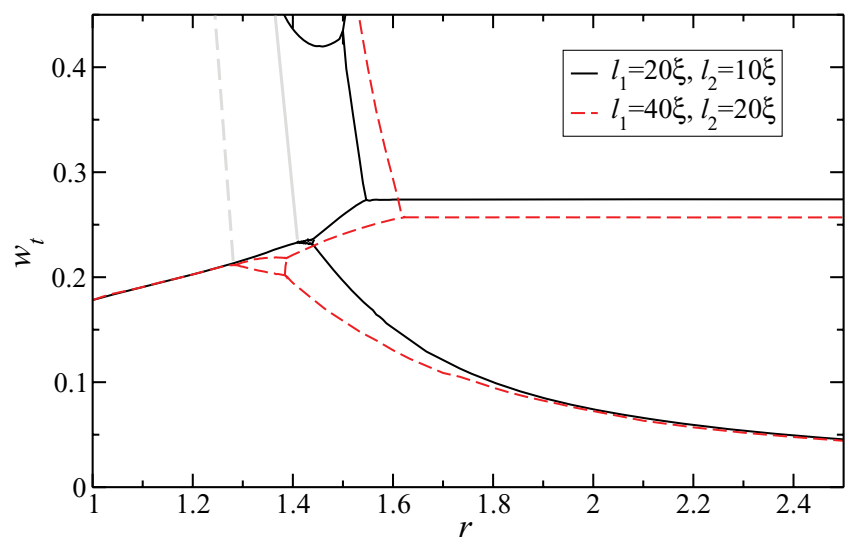

FIG. 11. (Color online) Phase diagrams for $l_{1}=20 \xi, l_{2}=10 \xi$ and $l_{1}=40 \xi, l_{2}=20 \xi$. The grey lines represent the weak first-order transition between the two distorted wet states (see Fig. 4).

smaller systems, while the bent filled states-with nematic droplets - are driven to a region of surface anchoring that is outside the wetting range of parameters for large systems. The re-entrant region involves unbent (rather than bent) filled states.

\section{CONCLUSION}

Simple fluids at crenellated surfaces exhibit a continuous filling transition where the interface unbends, followed by a wetting transition where the interface unbinds [5]. Nematic elasticity and topological defects, not present in simple fluids, lead to a variety of novel filled and wet states, characterized by distinct nematic textures [8]. As the geometrical parameters of the surface-scale and symmetry-vary, the anchoring strength versus roughness surface phase diagrams exhibit complex behavior, with re-entrant filling, where the sequence dry-filled-wet-filled states is observed at fixed roughness, as the anchoring strength increases. This results from a delicate stability balance of the new surface phases_-bent and unbent, with and without nematic droplets-which are not observed in other systems.

As triangular surfaces, crenellated surfaces exhibit geometrical surface singularities, but their effect on the nematic wetting phase diagram is substantially different. At triangular surfaces, the nucleation of defects is unavoidable, even at low roughness, for sufficiently large anchoring strengths $w$ [20]. As a consequence, the nematic director is always distorted. Thus, the generalized Wenzel's law [Eq. (3)] describes the wetting transitions (the only transitions at triangular surfaces), if the logarithmic contribution to the elastic energy from the defects is taken into account. At crenellated surfaces, however, the nucleation of defects and even smooth distortions are avoidable, and are avoided, at low roughness. The wet state is then characterized by a nearly uniform director, perpendicular to the top and bottom surfaces of the crenels. The energy of the nearly uniform wet state is determined simply by the (anisotropic) interfacial tensions.

As a general conclusion, at low and high roughness, the phase diagram of crenellated surfaces is similar to that of sinusoidal surfaces: there is a transition from dry-to-wet states at low roughness and from dry-to-filled states at high roughness. As in the sinusoidal case, the equilibrium configurations are those where, whenever possible, topological defects are avoided [22].

At intermediate roughness, however, crenellated surfaces exhibit the simple-fluid sequence, dry $\rightarrow$ filled $\rightarrow$ wet. As the anchoring strength increases further, the surface exhibits a de-wetting transition from the wet to a second filled state, where pinning of the NI interface is energetically favourable, when compared to a wet state where topological defects are nucleated at the top corners of the crenels. This re-entrant filling behavior is robust and similar diagrams were obtained for crenellated surfaces characterized by different geometrical parameters. Re-entrant filling has not been observed in simple fluids at crenellated surfaces nor in nematics at other structured surfaces.

In the intermediate roughness regime, different wet states co-exist: the symmetric wet state becomes asymmetrically distorted, as the deformations induced by the surface of the crenels increase and topological defects are nucleated at the corners of the crenelations. In addition, two filled states co-exist: the unbent filled state, where the NI interface is flat and pinned at the top corners of the crenels, is symmetrically distorted with a bent NI interface inside the crenels, even in wide crenels. In macroscopic crenels these bent interfaces will eventually unbend. However, we have found stable bent filled states, in a wide range of parameters, for geometries 30 to 40 times larger than $\xi$. When the system changes from a dry state to the bent filled state, a filling transition occurs but there is no unbending transition. These transitions were found to occur simultaneously, in all other systems, but are clearly separated for nematics at crenellated surfaces.

There are important questions concerning the dynamics of the transitions. We recall that the wetting and filling transitions reported here are first order and the understanding of the dynamics, which may be rather complex involving vortices near the corners of the crenels, as well as the effect of external fields are essential to investigate switching mechanisms among the nematic surface states. In future work Lattice Boltzmann simulations will be used to start addressing these questions [32].

We have studied the wetting behavior of nematics at triangular [20,21], sinusoidal [22], and crenellated surfaces. We have revealed common features of the phase diagrams of these structured surfaces and established a new rationale as well as simple rules for nematic wetting. Recently, we have developed a methodology to obtain the elastic contribution to the free energy based on Berreman's approach [33], valid even when defects are nucleated [34]. Extending this to a more general description of structured surfaces does not seem straightforward, but could lead to important generic results. It is now crucial to test these results with experiments.

\section{ACKNOWLEDGMENTS}

We gratefully acknowledge the financial support from the Portuguese Foundation for Science and Technology (FCT) through Grants No. PEst-OE/FIS/UI0618/2011, No. PTDC/FIS/098254/2008, and SFRH/BPD/40327/2007 (N.M.S.). J.M.R.-E. also acknowledges financial support from the Spanish MICINN through Grant No. FIS2009-09326, and Junta de Andalucía through Grant No. P09-FQM-4938, both 
co-funded by the EU FEDER. We would like to thank M. Blow and N. R. Bernardino for very fruitful discussions.

\section{APPENDIX A: CALCULATION OF $\sigma_{n s}^{\|}-\sigma_{n s}^{\perp}$ AND $\sigma_{n s}^{\perp}-\sigma_{i s}$}

Here we calculate the first two terms of Eq. (16). The calculation of $\sigma_{n s}^{\perp}$ and $\sigma_{n s}^{\|}$considers a planar surface, with preferred homeotropic anchoring, wet by a nematic with perpendicular or parallel orientation at the surface. The nematic is assumed to be aligned uniformly and the scalar order parameter is a function of the distance to the surface, $q(y)$, which can be described by the ansatz

$$
q_{\perp / \|}(y)=q_{b}+q_{o}^{\perp / \|} \exp \left(-y / y_{o}^{\perp / \|}\right),
$$

where $q_{b}$ is the bulk equilibrium scalar order parameter. At the coexistence temperature $q_{b}=1$. The parameters $q_{o}$ and $y_{o}$ depend on the orientation at the surface and are obtained by minimizing the free energy. $q_{o}+q_{b}$ is the degree of orientational order at the surface. $y_{o}$ is the length that characterizes the decay of the surface induced order.

In the perpendicular case the nematic director is parallel to the $y$ axis and the tensor order parameter is

$$
\underline{\underline{Q}}^{\perp}=q_{\perp}(y)\left(\begin{array}{ccc}
-1 / 2 & 0 & 0 \\
0 & 1 & 0 \\
0 & 0 & -1 / 2
\end{array}\right),
$$

while in the parallel case the tensor is

$$
\underline{\underline{Q}}^{\|}=q_{\|}(y)\left(\begin{array}{ccc}
1 & 0 & 0 \\
0 & -1 / 2 & 0 \\
0 & 0 & -1 / 2
\end{array}\right) .
$$

The bulk free-energy density is simply

$$
f_{b}^{\perp / \|}=q_{\perp / \|}^{2}(y)-2 q_{\perp / \|}^{3}(y)+q_{\perp / \|}^{4}(y) .
$$

In the two-elastic constant approximation, the elastic freeenergy density for the perpendicular and the parallel cases are, respectively,

$$
\begin{gathered}
f_{e}^{\perp}=\frac{1}{2}\left(\frac{d}{d y} q_{\perp}(y)\right)^{2}, \\
f_{e}^{\|}=\frac{1}{2}\left(\frac{3+k / 2}{3+2 k}\right)\left(\frac{d}{d y} q_{\|}(y)\right)^{2} .
\end{gathered}
$$

At the surface, the preferred tensor order parameter is

$$
\underline{\underline{Q}} s=q_{s}(y)\left(\begin{array}{ccc}
-1 / 2 & 0 & 0 \\
0 & 1 & 0 \\
0 & 0 & -1 / 2
\end{array}\right),
$$

where we will take $q_{s}=q_{b}=1$. In the linear regime [Eq. (10)], valid for weak anchoring, the surface free-energy densities are

$$
\begin{gathered}
f_{s}^{\perp}=-w q_{\perp}(0), \\
f_{s}^{\|}=\frac{w}{2} q_{\|}(0) .
\end{gathered}
$$

Using the ansatz functions the free energies per unit area for the perpendicular and the parallel cases are, respectively,

$$
F^{\perp}=\frac{q_{o}^{\perp^{2}}}{2}\left(y_{o}^{\perp}+\frac{4 y_{o}^{\perp}}{3} q_{o}^{\perp}+\frac{y_{o}^{\perp}}{2} q_{o}^{\perp 2}+\frac{1}{2 y_{o}^{\perp}}\right)-w\left(q_{o}^{\perp}+1\right),
$$

$$
\begin{aligned}
F^{\|}= & \frac{q_{o}^{\|^{2}}}{2}\left(y_{o}^{\|}+\frac{4 y_{o}^{\|}}{3} q_{o}^{\|}+\frac{y_{o}^{\|}}{2} q_{o}^{\|^{2}}+\frac{3+k / 2}{3+2 k} \frac{1}{2 y_{o}^{\|}}\right) \\
& +\frac{w}{2}\left(q_{o}^{\|}+1\right) .
\end{aligned}
$$

Minimization with respect to $q_{o}$ and $y_{o}$, for weak anchoring, results in

$$
\begin{aligned}
F^{\perp} & =-w+O\left(w^{2}\right), \\
F^{\|} & =\frac{w}{2}+O\left(w^{2}\right) .
\end{aligned}
$$

And thus, we find $\sigma_{n s}^{\|}-\sigma_{n s}^{\perp}=\frac{3}{2} w+O\left(w^{2}\right)$. The calculation of $\sigma_{i s}$ proceeds in a similar fashion by considering that the surface is wet by an isotropic phase, and we find $\sigma_{n s}^{\perp}-\sigma_{i s}=$ $-w+O\left(w^{3}\right)$, as calculated in [20].

\section{APPENDIX B: INTERFACIAL PROFILE OF A NEMATIC DROPLET PINNED TO A PLANAR SURFACE AT COMPLETE WETTING}

We estimate the shape and surface excess free energy of a nematic droplet in contact with a planar surface under complete wetting conditions, with the interface pinned along two parallel straight lines along the surface, at $x=0$ and $x=l$. We assume homeotropic anchoring at the surface and a thin droplet. Then the gradients of the interfacial height profile may be assumed small and the nematic orientation uniform. Taking into account the isomorphism between the Landau-Ginzburg and the Landau-de Gennes under these conditions [8], and that interfacial Hamiltonians are a good representation of the interfacial behavior of simple fluids in contact with structureless surfaces [35], we can estimate the free energy of the droplet per unit length in the $y$ direction as

$$
\begin{aligned}
f_{\text {drop }}= & \sigma_{n i}^{\perp} \int_{0}^{l}\left[1+\frac{1}{2}\left(\frac{d z}{d x}\right)^{2}\right] d x+\sigma_{n s}^{\perp} l \\
& +\int_{0}^{l} w e^{-\sqrt{2} z(x) / \xi} d x,
\end{aligned}
$$

where $w>0$ plays the role of an effective Hamaker constant. The Euler-Lagrange equation associated to the functional Eq. (B1) is

$$
\sigma_{n i}^{\perp} \frac{d^{2} z}{d x^{2}}=-\frac{\sqrt{2} w}{\xi} e^{-\sqrt{2} z(x) / \xi} .
$$

Multiplying Eq. (B2) by $d z / d x$ and integrating with respect to $z$, we find

$$
\frac{\sigma_{n i}^{\perp}}{2}\left(\frac{d z}{d x}\right)^{2}-w e^{-\sqrt{2} z(x) / \xi}=-w e^{-\sqrt{2} z(l / 2) / \xi} .
$$

The droplet interfacial profile is then given by

$$
\frac{d z}{d x}=-\frac{x-l / 2}{|x-l / 2|} \sqrt{\frac{2 w}{\sigma_{n i}^{\perp}}\left(e^{-\sqrt{2} z(x) / \xi}-e^{-\sqrt{2} z(l / 2) / \xi}\right)},
$$


which is solved by standard methods, yielding

$$
z(x)=z(l / 2)+\sqrt{2} \xi \ln \cos \left(\sqrt{\frac{w}{\sigma_{n i}^{\perp} \xi^{2}}} e^{-\sqrt{2} z(l / 2) / 2 \xi}|x-l / 2|\right) .
$$

In order to calculate the maximum interfacial height $z(l / 2)$, we impose the pinning condition $z(0)=z(l)=0$. We define

$$
\lambda=\sqrt{\frac{w}{\sigma_{n i}^{\perp} \xi^{2}}} e^{-\sqrt{2} z(l / 2) / 2 \xi} l / 2
$$

which satisfies the transcendental equation

$$
\frac{2 \lambda}{l} \sqrt{\frac{w}{\sigma_{n i}^{\perp} \xi^{2}}}=\cos \lambda
$$

solved by graphical methods. For large $l$, the solution is close to $\pi / 2$. Expand the cosine function around this value, the solution up to terms of order of $l^{-2}$, is

$$
\lambda \approx \frac{\pi}{2}\left(1-\frac{2}{l} \sqrt{\frac{w}{\sigma_{n i}^{\perp} \xi^{2}}}\right) .
$$

Substituting Eq. (B8) into Eq. (B5), we obtain the asymptotic interfacial profile, away from the pinning sites,

$$
z(x) \approx \sqrt{2} \xi \ln \left(\frac{l}{\pi} \sqrt{\frac{w}{\sigma_{n i}^{\perp} \xi^{2}}} \sin \frac{\pi x}{l}\right),
$$

in agreement with the result obtained by applying conformal techniques to droplet shapes for simple fluids [36].

Substitution of Eq. (B5) into Eq. (B1) yields the equilibrium free energy of the droplet per unit length in the $y$ direction,

$$
f_{\text {drop }}^{\text {equilibrium }}=\left(\sigma_{n i}^{\perp}+\sigma_{n s}^{\perp}\right) l+\frac{8 \sigma_{n i}^{\perp} \xi^{2}}{l}\left(\lambda \tan \lambda-\lambda^{2} / 2\right) .
$$

For large $l$, we can use Eq. (B8) in Eq. (B10), yielding the asymptotic behavior,

$$
f_{\text {drop }}^{\text {equilibrium }} \approx\left(\sigma_{n i}^{\perp}+\sigma_{n s}^{\perp}\right) l+4 \sqrt{w \sigma_{n i}^{\perp} \xi^{2}}+\mathcal{O}\left(l^{-1}\right) .
$$

[1] D. E. Sullivan and M. M. Telo da Gama, in Fluid Interfacial Phenomena, edited by C. A. Croxton (Wiley, New York, 1986), p. 45.

[2] S. Dietrich, in Phase Transitions and Critical Phenomena, edited by C. Domb and J. Lebowitz (Academic, New York, London, 1988), Vol. 12.

[3] M. Schick, in Liquids at Interfaces, edited by J. Charvolin, J. F. Joanny, and J. Zinn-Justin (Elsevier, Amsterdam, 1990).

[4] D. Bonn, J. Eggers, J. Indekeu, J. Meunier, and E. Rolley, Rev. Mod. Phys. 81, 739 (2009).

[5] C. Rascón, A. O. Parry, and A. Sartori, Phys. Rev. E 59, 5697 (1999).

[6] C. Rascón and A. O. Parry, Nature (London) 407, 986 (2000).

[7] D. Quéré, Annu. Rev. Mater. Res. 38, 71 (2008).

[8] P. Patricio, J. M. Romero-Enrique, N. M. Silvestre, N. R. Bernardino, and M. M. Telo da Gama, Mol. Phys. 109, 1067 (2011).

[9] P. Sheng, Phys. Rev. Lett. 37, 1059 (1976).

[10] P. Sheng, Phys. Rev. A 26, 1610 (1982).

[11] A. K. Sen and D. E. Sullivan, Phys. Rev. A 35, 1391 (1987).

[12] F. N. Braun, T. J. Sluckin, and E. Velasco, J. Phys.: Condens. Matter 8, 2741 (1996).

[13] H. Yokoyama, S. Kobayashi, and H. Kamei, Mol. Cryst. Liq. Cryst. 99, 39 (1983).

[14] H. Hsiung, T. Rasing, and Y. R. Shen, Phys. Rev. Lett. 57, 3065 (1986).

[15] H. Hsiung, T. Rasing, and Y. R. Shen, Phys. Rev. Lett. 59, 1983 (1987).

[16] W. Chen, L. J. Martinez-Miranda, H. Hsiung, and Y. R. Shen, Phys. Rev. Lett. 62, 1860 (1989).

[17] B. Alkhairalla, H. Allinson, N. Boden, S. D. Evans, and J. R. Henderson, Phys. Rev. E 59, 3033 (1999).
[18] L. Harnau, F. Penna, and S. Dietrich, Phys. Rev. E 70, 021505 (2004)

[19] J. P. Bramble, S. D. Evans, J. R. Henderson, C. Anquetil, D. J. Cleaver, and N. J. Smith, Liq. Cryst. 34, 1059 (2007).

[20] P. Patrício, C. T. Pham, and J. M. Romero-Enrique, Eur. Phys. J. E 26, 97 (2008).

[21] J. M. Romero-Enrique, C. T. Pham, and P. Patrício, Phys. Rev. E 82, 011707 (2010).

[22] P. Patricio, N. M. Silvestre, C.. T. Pham, and J. M. RomeroEnrique, Phys. Rev. E 84, 021701 (2011).

[23] C. V. Brown, M. J. Towler, V. C. Hui, and G. P. Bryan-Brown, Liq. Cryst. 27, 233 (2000).

[24] C. Uche, S. J. Elston, and L. A. Parry-Jones, J. Phys. D 38, 2283 (2005).

[25] A. O. Parry, C. Rascón, N. B. Wilding, and R. Evans, Phys. Rev. Lett. 98, 226101 (2007).

[26] K. Rejmer and M. Napiórkowski, Phys. Rev. E 62, 588 (2000).

[27] G. Barbero, Lett. Nuovo Cimento Soc. Ital. Fis. 29, 553 (1980).

[28] P. Patricio, M. M. Telo da Gama, and S. Dietrich, Phys. Rev. Lett. 88, 245502 (2002).

[29] T. Ohzono, and J.-I. Fukuda, Nat. Commun. 3, 701 (2012).

[30] P. G. de Gennes and J. Prost, The Physics of Liquid Crystals, 2nd ed. (Clarendon, Oxford, 1993).

[31] D. Andrienko, M. Tasinkevych, P. Patrício, and M. M. Telo da Gama, Phys. Rev. E 69, 021706 (2004).

[32] M. Blow (private communication).

[33] D. W. Berreman, Phys. Rev. Lett. 28, 1683 (1972).

[34] O. A. Rojas-Gomez, and J. M. Romero-Enrique, arXiv:1205.2906.

[35] E. Brézin, B. I. Halperin, and S. Leibler, J. Phys. 44, 775 (1983).

[36] A. O. Parry, E. D. Macdonald, and C. Rascón, J. Phys.: Condens. Matter 13, 383 (2001). 